\title{
Statistical approaches to computing sample size in cluster randomized trials: a simulation study
}

\section{Ashutosh Ranjan}

IQVIA

\section{Guangzi Song}

Drexel University School of Public Health

\section{Christopher S Coffey}

University of lowa

Leslie A McClure ( $\sim$ lam439@drexel.edu )

Drexel University School of Public Health https://orcid.org/0000-0002-2465-6739

\section{Research article}

Keywords: Cluster-randomized trials, unequal clusters, coefficient of variation, weighted analysis, type I error, power

Posted Date: June 3rd, 2020

DOI: https://doi.org/10.21203/rs.2.18978/v2

License: (1) (1) This work is licensed under a Creative Commons Attribution 4.0 International License. Read Full License 
Statistical approaches to computing sample size in cluster randomized trials: a simulation study Short Title: Sample size calculations for CRTs

Author 1: Ranjan, Ashutosh

IQVIA

Durham, NC, USA

Author 2: Song, Guangzi

Dornsife School of Public Health,

Drexel University, Philadelphia, PA, USA

Author 3: Coffey, Christopher S.

Department of Biostatistics, College of Public Health,

University of Iowa, Iowa City, IA, USA

Author 4: McClure, Leslie A.*

Dornsife School of Public Health,

Drexel University, Philadelphia, PA, USA

*Corresponding author 


\section{ABSTRACT}

Background: Cluster randomized trials, which randomize groups of individuals to an intervention, are common in health services research when one wants to evaluate improvement in a subject's outcome by intervening at an organizational level. For many such trials, sample size calculation is performed under the assumption of equal cluster size. For a variety of reasons, many trials that set out to recruit clusters of the same size end up with unequal clusters. This leads to a misalignment between the method used for sample size calculation and the data analysis, which may affect trial power. Various weighted analysis methods for analyzing cluster means have been suggested to overcome the problem introduced by unbalanced clusters; however, the performance of such methods has not been evaluated extensively.

Methods: We examine the use of the general linear model for analysis of clustered randomized trials that assume equal cluster sizes during the planning stage, but for which the realized cluster sizes are unequal. We demonstrate the performance of three approaches using different weights for analyzing the cluster means: (1) the standard analysis of cluster means, (2) weighting by cluster size, and (3) minimum variance weights. Several distributions are used to generate cluster sizes to assess a range of patterns of imbalance. The variability in cluster size is measured by the coefficient of variation (CV). We assess the impact of using each of the three methods of analysis with respect to type I error and power of the study and how each are impacted by the variability in cluster size via simulations.

Results: Analyses that assumes equal clusters provide a reasonable approximation when cluster sizes vary minimally $(\mathrm{CV}<0.30)$. For analyses weighted by cluster size type I errors were inflated, and that worsened as the variation in cluster size increases, despite reasonable power. However, minimum variance weighted analyses best maintain target power and level of significance under scenarios considered.

Conclusion: Unweighted analyses work well as an approximate method when the variation in cluster size is minimal. However, using minimum variance weights performs much better across the full range of variation in cluster size and is recommended. 
Keywords: Cluster-randomized trials, unequal clusters, coefficient of variation, weighted analysis, type I error, power

\section{BACKGROUND}

Importance of cluster-randomized trials

A cluster-randomized trial (CRT) is a trial where groups of individuals are randomized to different interventions. These designs are commonplace in health care research(1), where they are increasingly being used to assess the effectiveness of interventions against infectious diseases as well as for prevention and treatment of non-communicable diseases.(2, 3) Findings from such studies have the potential to help government agencies formulate health policies.

In a CRT, the responses of individuals within a cluster tend be correlated, and that correlation is measured by the intra-cluster correlation (ICC). The correlated responses preclude the use of classical methods of inference based on the assumption of independence.

\section{Sample Size Calculations in CRTs}

Compared to an individually randomized trial, sample size in a cluster-randomized setting is a function of the number of clusters, cluster size, and ICC, in addition to the other necessary parameters. When the cluster sizes are equal, the simple sample size calculation based on the assumption of equal cluster sizes or using the mean cluster size are based on an analytic approach that allows exact inference.(4) However, in reality, cluster sizes are often unequal even for trials that planned for equal clusters. In fact, a review of 200 CRTs found that the recruitment strategy had led to unequal clusters in two-thirds of the trials,(5) and unequal cluster 
sizes affect trial power when the sample size is estimated under the assumption of equal cluster sizes, with power decreasing as the imbalance among the clusters increases.

To overcome the loss in trial power due to imbalance in cluster sizes, Manatunga et al.(6) used an asymptotic correction term based on the distribution of cluster size in the large sample formula to compute sample size provided by Donner et al.(4) A practical difficulty in using such an approach is determining the distribution of cluster size before the trial has begun. Further, in their simulation study, they only consider values of ICC greater than 0.25 and do not discuss the impact of their proposed correction on the type I error of the trial.

Eldridge et al.(5) suggested incorporating the coefficient of variation (CV) of the cluster size in the sample size formula and show the utility of the proposed approach using a hypothetical example. However, accurate estimates of $\mathrm{CV}$ may not be easy to obtain, and the appropriateness of this method also depends on the ICC being accurate. Van Breukelen and colleagues have provide a number of approaches, including using relative efficiency of unequal versus equal cluster sizes, deriving an approximate formula based on CV of cluster size and the ICC, all of which assume known values for these parameters. (7-9) While these authors provide suggestions for how to choose an ICC if one cannot be derived from prior studies, their result still relies on researchers' ability to determine a reasonable range for the ICC.(8) More recently, You et al.(10) also proposed a relative efficiency measure based on the non-centrality parameter. Once again, this method require prior knowledge of the $\mathrm{CV}$ of cluster size, which may not be known at the planning stage. Thus, under the premise of the inability to obtain accurate estimates of $\mathrm{CV}$, and unclear knowledge of the distribution of cluster size, the simplest approach available to clinical 
researchers for sample size calculation in cluster randomized trials is to assume of equal cluster size.

\section{Analytical methods for CRTs}

When the unequal cluster size is unable to be accounted for in the planning, it is necessary to take it into account in the analysis stage. Three commonly employed analytic methods for data arising from a CRT are: (a) analysis of cluster means using classical methods, (b) linear mixed effects model and (c) generalized estimating equations.(11) While more complex models (multivariate methods, mixed models, and GEEs) are popular for analyzing the data, respective methods for power analysis have not found such universal applicability.(12) Therefore, in this paper, we focus only on the first analysis approach so that the power and data analysis are aligned under the generalized linear model (GLM) framework.

When the cluster sizes are equal, using classical methods for analyzing the cluster means allows use of all the methods of classical theory.(13) However, this Exact Under Balanced Design (EUBD) approach only provides approximate results in the presence of unequal clusters. The EUBD approach provides a unified setup for power and data analysis under the framework of GLM and in doing so makes all commercial/free software capable of handling linear models available for the design and analysis of CRTs with unequal cluster sizes.

As described above, CRT designed assuming equal cluster sizes are underpowered in the presence of unequal clusters. A weighted analysis of cluster mean has been suggested to offset the loss in power due to the unequal clusters. $(5,14)$ Kerry and Bland(14) compared different 
weighting schemes and found that minimum variance weights, proportional to the inverse of the variances of cluster means, were the best in terms of achieving target power, but do not discuss the implications of different weights on the type I error, although Johnsen et al (2015) provide a thorough discussion of type I error, without discussion of power(15). Guittet et al.(16) used these weights to study "Pareto" type imbalance where $80 \%$ of subjects belong to only $20 \%$ of clusters, although many CRTs may not necessarily show imbalance of this nature.

In general, within the approach of analyzing cluster means under the generalized linear model framework, there are two ways to analyze the data - simple unweighted analysis of cluster means, and weighted analysis of cluster means. Johnson et al showed that type I error was wellcontrolled for a variety of weighting approaches and recommended different weighting approaches depending on the number of clusters per $\operatorname{arm}(15)$.

Aligning the sample size calculation and analysis approach

In general, one would like to align the sample size calculation and analytic approach for best operating characteristics. The EUBD approach allows this alignment and provides exact inference as long as equal cluster sizes are expected during the design stage and equal clusters are, in fact, observed during recruitment stage. For cases where a defensible estimate of $\mathrm{CV}$ of cluster size is available at the design stage, methods exist to compute the sample size that also aligns the calculation with the analysis.

However, there are scenarios where alignment of the sample size estimation and analytic approach may not be practical. For example, equal clusters may be expected at the design stage, 
but the study results in unequal clusters. Further, unequal clusters may be expected but there may not be reliable estimates of $\mathrm{CV}$ of cluster size available upon which base the sample size calculation; that is, no prior information was available from previous trials. Under such circumstances, researchers often compute sample size based on equal clusters, while the analysis takes into account the unequal clusters. This introduces a misalignment between the sample size calculation and the data analysis approach and affects the trial power adversely.

\section{Objective and outline}

Our objective is to evaluate the impact on power and type I error of computing the sample size calculation assuming equal cluster sizes, when the trial in fact captures unequal clusters, through three analytic approaches using different weights, under a range of scenarios.

We will first assess a standard analysis of cluster means. We will then perform a weighted analysis of cluster means using cluster size weights and minimum variance weights. Based on our results, we make recommendations on suitability of approaches under different conditions.

In Section 2, we describe the model of a CRT with unequal cluster sizes that will be used for generating data and the univariate model associated with a balanced cluster trial for a two-group

parallel study. Details about the simulation study are given in Section 3. We present the results of the simulation in Section 4, followed by discussion of results in Section 5.

\section{METHODS}

Model Statement and Sample Size Calculation 
A linear mixed model for $p_{g i}$ - correlated observations on a cluster $i \in\left\{1, \ldots, N_{2}\right\}$ assigned to intervention $g \quad\{1, \ldots, G\}$ may be written as

$$
\boldsymbol{y}_{i}=\boldsymbol{X}_{i} \boldsymbol{\beta}+\boldsymbol{z}_{i} d_{i}+\boldsymbol{e}_{i}
$$

where the $G \times 1$ vector $\boldsymbol{\beta}$ contains unknown parameters, while the $p_{g i} \times G$ matrix $\boldsymbol{X}_{i}$ is the known design matrix of fixed effects. The $p_{g i} \times 1$ vector $z_{i}$ is the design matrix for the clusterspecific random effect. Assume $d_{i} \sim \mathcal{N}\left(0, \sigma_{b}^{2}\right)$ mutually independent of the residual error component $\boldsymbol{e}_{i} \sim \mathcal{N}_{p_{g i}}\left(\mathbf{0}, \sigma_{e}^{2} \boldsymbol{I}_{p_{g i}}\right)$. With just one random effect for cluster, $\boldsymbol{z}_{i} \equiv \mathbf{1}_{p_{g i}}$ and therefore, $\boldsymbol{\Sigma}_{i}=\sigma_{b}^{2} \mathbf{1}_{p_{g i}} \mathbf{1}_{p_{g i}}^{\prime}+\sigma_{e}^{2} \boldsymbol{I}_{p_{g i}}$ leading to $\boldsymbol{\Sigma}_{i}=\sigma^{2}\left[\mathbf{1}_{p_{g i}} \mathbf{1}_{p_{g i}}^{\prime} \rho+\boldsymbol{I}_{p_{g i}}(1-\rho)\right]$ as the covariance matrix for $i^{t h}$ cluster.

Assuming $p_{g i}=N_{1}$ for a trial with equal clusters and applying a suitable transformation, as described in Gurka et al. (2007), the linear mixed model can be written as a univariate linear model for the test of fixed intervention effect, given by (2.2).

$$
\boldsymbol{y}_{s}=\boldsymbol{X}_{s} \boldsymbol{\beta}+\boldsymbol{e}_{s}
$$

In equation (2.2), $y_{s}$ is the cluster mean and the covariance matrix is given by $\boldsymbol{\Sigma}_{s}=N_{1}^{-1} \sigma^{2}\left\{1+\left(N_{1}-1\right) \rho\right\}$. Use of cluster mean to test the hypothesis of no difference in intervention groups i.e. $H_{0}: \delta=0$ against $H_{1}: \delta \neq 0$ allows using the theory of Student's $t$-test to compare two independent groups. This also allows using (2.3) for computation of sample size for comparing two means, i.e. testing $H_{0}: \mu_{1}=\mu_{2}$ against $H_{1}: \mu_{1} \neq \mu_{2}$. 


$$
N_{2} N_{1}=\frac{2\left(t_{\alpha / 2,2\left(N_{2}-1\right)}+t_{\beta, 2\left(N_{2}-1\right)}\right)^{2} \sigma^{2}\left[1+\left(N_{1}-1\right) \rho\right]}{\left(\mu_{1}-\mu_{2}\right)^{2}}
$$

In (2.3), $\mu_{1}$ and $\mu_{2}$ are the means of the treatment and control groups, respectively. Assuming that the response variable has variance $\sigma^{2}$ and that $N_{2}$ clusters of $N_{1}$ subjects are assigned to each of the two groups, a total of $N_{2} N_{1}$ subjects across all clusters are needed when the ICC is $\rho$. The critical values of a $t$-distribution depend on the degree of freedom for the test and therefore the formula may require two or more iterations,(17) however, this complexity is easily addressed by using free or commercial software programs.

When cluster sizes vary, no exact method exists (see Appendix) and the transformation leads to a GLM with heteroscedastic errors where the covariance matrix is of the form $\boldsymbol{\Sigma}_{s}=\sigma^{2} \boldsymbol{D}$ where $\boldsymbol{D}$ is a positive definite matrix. The elements of $\boldsymbol{D}$ are a function of the ICC and the size of cluster. In this paper, we consider three analyses based on the structure of the $\boldsymbol{D}$ matrix. We present them below in Table 1.

Table 1. Structure of $D$ for different analyses

Analysis $\quad$ Structure of $\boldsymbol{D}$ matrix
Analyzing cluster means ignoring

$$
\boldsymbol{D}=\boldsymbol{I}_{g N_{2}}(\text { identity matrix })
$$
the variability in cluster size
Analyzing cluster means weighted

$$
\boldsymbol{D}=\operatorname{Diag}\left(1 / p_{g 1}, \ldots, 1 / p_{g N_{2}}\right)
$$
by cluster size 
Analyzing cluster means weighted by minimum variance weight

$$
\boldsymbol{D}=\operatorname{Diag}\left(\left[1+\left(p_{g 1}-1\right) \rho\right] / p_{g 1}, \ldots,\left[1+\left(p_{g N_{2}}-1\right) \rho\right] / p_{g N_{2}}\right)
$$

As such, in this paper, we investigate the performance of cluster level analysis that allows us to make use of a $t$-test to compare two independent samples. The first method uses a two-sample ttest on cluster means ignoring the variability in cluster sizes. The second and third approach use a weighted $t$-test on cluster means where means are weighted by cluster size and minimum variance weights, respectively. When cluster size is used as weights, more weight is given to clusters with more individuals. Minimum variance weights are proportional to the inverse of the variances of cluster means. With small cluster sizes and small ICC these weights are equivalent to the cluster size weights (see Appendix).

\section{Overview of Simulation Study}

Monte Carlo simulations were used to examine the effect of imbalance in cluster sizes on power and type I error for the test of an intervention effect, i.e. $H_{0}: \delta=0$. A $6 \times 4 \times 2 \times 6$ factorial plan was used for the simulation that considered 6 different cluster size distributions (discussed below); 4 values of ICC: $\rho \in(0.001,0.01,0.1,0.3) ; 2$ values of the treatment difference: $\delta \in(0.25,0.50) ;$ and 6 values of mean cluster size: $\bar{N}_{1} \in(8,16,32,64,128,256)$ yielding a total of 288 different combinations of design parameters. For the sake of convenience, the total variance $\sigma^{2}\left(=\sigma_{w}^{2}+\sigma_{b}^{2}\right)$ was set equal to 1 . This restriction allowed defining $\sigma_{b}^{2}=\rho$ and $\sigma_{w}^{2}=1-\rho$. The value of $\alpha$ was fixed at 0.05 . Using the expression given in (2.3), the number 
of independent clusters $\left(N_{2}\right)$ assuming equal cluster size was computed for $80 \%$ target power using the GLMPOWER procedure in SAS 9.2 software.

In the absence of published ranges of variability of cluster sizes in the literature, several distributions were used to generate the size of clusters providing different levels of variability in cluster sizes. For all the distributions, we fixed minimum and maximum cluster sizes at 1 and $2 \times N_{1}$, respectively. The parameters used for different distributions are presented in Table 2 . with the cluster sizes from the uniform distribution being the most variable and those from the Poisson distribution least variable. Cluster sizes from bimodal, Gaussian, negative skewed and triangular distribution fell in between the two in decreasing order of magnitude.

Table 2. Parameters of various distributions to generate cluster sizes

\begin{tabular}{|c|c|c|c|c|}
\hline Distribution & Minimum & Maximum & Mean & Std. Deviation \\
\hline Uniform & 1 & $2 \times N_{1}$ & & \\
\hline Poisson & & & $N_{1}$ & \\
\hline Triangular & 1 & $2 \times N_{1}$ & Mode $: N_{1}$ & \\
\hline Gaussian & & & {$\left[1+\left(2 \times N_{1}\right)\right] / 2$} & {$\left[\left(2 \times N_{1}\right)-1\right] / 4$} \\
\hline $\begin{array}{l}\text { Negative Skew Gaussian } \\
\text { (truncated at } N_{1} \text { ) }\end{array}$ & & & {$\left[1+\left(2 \times N_{1}\right)\right] / 2$} & {$\left[\left(2 \times N_{1}\right)-1\right] / 4$} \\
\hline Bimodal & & & $\begin{array}{l}\text { Mean } 1: N_{1}-0.5 N_{1} \\
\text { Mean } 2: N_{1}+0.5 N_{1}\end{array}$ & $\left(\mu_{2}-\mu_{1}\right) / 4$ \\
\hline
\end{tabular}


For all 288 combinations of the design parameters, 1000 and 5000 replications of data were simulated for power and type I error, respectively. The two-sample $t$-test ignoring the cluster size variation and the weighted $t$-test were used to analyze the transformed data. Power and empirical type I error were then computed as the proportion of trials where the p-value was smaller than

0.05 when $\delta=\hat{\delta}$ (power) and $\delta=0$ (type I error). Type I error values obtained were considered equal to the nominal level if they fell in the interval $(0.047,0.053)$. Similarly, power in the interval $(0.79,0.81)$ was assumed to meet target level.

Since the main aim of conducting this simulation study was to study the performance of methods in the presence of unbalanced clusters, the results should be interpreted from the point of view of cluster size variation. To achieve this, the results are presented in the context of CV of cluster sizes. We categorized the $\mathrm{CV}$ into 3 classes - low variation $\left(\begin{array}{ll}C V & 0.30\end{array}\right)$, moderate variation $(0.30<C V \quad 0.50)$ and high variation $(C V>0.50)$ Out of the 288 combinations, $128(\sim 45 \%)$ fall into the 'high variation' category, while $58(\sim 20 \%)$ fall into the 'low variation' category. The remaining $102(\sim 35 \%)$ are in the moderate variation category.

\section{RESULTS}

Performance of the three methods to analyze the data

Summary statistics for observed type I error and power are presented in Table 3, including the minimum, maximum, mean and standard deviation for type I error and power values obtained under different scenarios of data analysis for the three different categories of variation in cluster size. We also present the percentage of cases where the type I error is inflated, and the power is below target level. Under the EUBD approach to sample size calculation, both the weighted 
approaches perform similarly in achieving target power. The choice of method for data analysis does not make a substantive difference when the $\mathrm{CV}$ of cluster sizes is less than 0.30 . However, the performance of weighted analysis using cluster size as weights declines as the CV of the cluster size increases. Average type I error was found to be inflated up to 0.073 (s.d. $=0.019$ ) when the $\mathrm{CV}$ of cluster size was greater than 0.50 , while the proportion of underpowered tests increases.

Table 3. Summary statistics for empirical type I error $(\alpha)$ and power $(1-\beta)$ attained by coefficient of variation (CV) of cluster sizes

\begin{tabular}{|c|c|c|c|c|c|c|c|c|c|c|c|}
\hline \multirow[b]{2}{*}{$\mathrm{CV}$} & \multirow[b]{2}{*}{ Weight } & \multicolumn{5}{|c|}{ Type I Error } & \multicolumn{5}{|c|}{ Power } \\
\hline & & Min. & Max. & Mean & $\mathrm{SD}$ & $\begin{array}{c}\% \\
\text { Cases } \\
\text { Inflated }\end{array}$ & Min. & Max. & Mean & $\mathrm{SD}$ & $\begin{array}{c}\% \text { Cases } \\
\text { under- } \\
\text { powered }\end{array}$ \\
\hline \multirow{3}{*}{$\begin{array}{l}\mathrm{CV} \leq \\
0.30\end{array}$} & None & 0.042 & 0.055 & 0.050 & 0.003 & 17 & 0.69 & 1.00 & 0.83 & 0.06 & 22 \\
\hline & $\begin{array}{l}\text { Cluster } \\
\text { Size }\end{array}$ & 0.044 & 0.065 & 0.052 & 0.004 & 24 & 0.73 & 1.00 & 0.84 & 0.06 & 12 \\
\hline & $\begin{array}{l}\text { Min. } \\
\text { Variance }\end{array}$ & 0.042 & 0.059 & 0.050 & 0.003 & 21 & 0.73 & 1.00 & 0.84 & 0.06 & 10 \\
\hline \multirow{3}{*}{$\begin{array}{l}0.30< \\
\mathrm{CV} \\
\leq 0.50\end{array}$} & None & 0.038 & 0.062 & 0.050 & 0.005 & 23 & 0.45 & 1.00 & 0.76 & 0.10 & 65 \\
\hline & $\begin{array}{l}\text { Cluster } \\
\text { Size }\end{array}$ & 0.040 & 0.086 & 0.060 & 0.010 & 72 & 0.70 & 1.00 & 0.81 & 0.06 & 39 \\
\hline & $\begin{array}{l}\text { Min. } \\
\text { Variance }\end{array}$ & 0.039 & 0.063 & 0.051 & 0.005 & 32 & 0.70 & 1.00 & 0.81 & 0.06 & 40 \\
\hline \multirow{3}{*}{$\begin{array}{l}\mathrm{CV}> \\
0.50\end{array}$} & None & 0.014 & 0.067 & 0.045 & 0.009 & 9 & 0.34 & 0.96 & 0.66 & 0.14 & 84 \\
\hline & $\begin{array}{l}\text { Cluster } \\
\text { Size }\end{array}$ & 0.044 & 0.128 & 0.073 & 0.019 & 85 & 0.65 & 1.00 & 0.81 & 0.06 & 45 \\
\hline & $\begin{array}{l}\text { Min. } \\
\text { Variance }\end{array}$ & 0.030 & 0.064 & 0.050 & 0.004 & 19 & 0.67 & 1.00 & 0.80 & 0.06 & 49 \\
\hline
\end{tabular}


In general, the simple analysis of cluster means assuming equal cluster sizes performs reasonably with respect to controlling the inflation in type I error compared to the other methods. However, it fails to maintain the target power as the variation in cluster size increases $(\mathrm{CV}>0.30)$. On the other hand, the method of weighting cluster means with the size of clusters does a better job of maintaining power at the target level, but may do so at a cost of inflated type I error. Nearly $85 \%$ of cases have an inflated type I error rate when analyzing the data using cluster size as weights with $\mathrm{CV}>0.50$, although average power is $81 \%$ ( $45 \%$ of simulations are underpowered). Finally, weighting cluster means by the minimum variance weight performs the best in terms of balancing the type I error and target power across the entire range of conditions. Since the simple analysis of cluster means provides comparable results to the other two methods when the variation in cluster size is low $(\mathrm{CV}<0.30)$, we do not discuss the scenario in further results for brevity.

Out of the 288 cases considered in simulation, approximately $45 \%(\sim 128)$ of the cases had a CV of cluster size greater than 0.50 . For these cases, cluster size weights led to an inflated type I error rate in $85 \%$ cases, but average power was $81 \%$, with $45 \%$ of simulations underpowered. The distribution of type I error and power for the three categories of CV is presented in Figure 1, showing that the use of a weighted method leads to better power than the standard analysis of cluster means when the $\mathrm{CV}$ is moderate to large.

Figure 1. Distribution of type I error (left) and power (right) for three categories of CV 
Figure 1 Legend: Distribution of type I error (left) and power (right) for the three categories of CV: (a) $C V \quad 0.30$; (b) $0.30<C V \quad 0.50$ and (c) $C V>0.50$. The reference line for nominal type I error and target power are shown by a dotted line at 0.05 and 0.80 respectively.

\section{Effect of ICC}

Three categories of ICC are studied - small ( $\rho=0.001$ or 0.01$)$, moderate $(\rho=0.1)$ and large ( $\rho=0.3$ ), similar to values of ICC that may be found in community trials or familial studies, for example.(18-22) Average type I error and power values obtained from simulation under different scenarios are presented in Table 4, which clearly indicate that the ICC can affect the power of a study to a much greater extent when an analysis assuming equal clusters is performed. For small ICC values $(\rho \leq 0.01)$ and large $\mathrm{CV}$ of cluster size $(>50 \%)$, the average power dropped to 0.59 with the standard analysis of cluster means. Using either of the weighted approaches maintains the study power at target levels, but cluster size weights leads to an inflated type I error (up to average of 0.086 when the $\mathrm{CV}>0.50$ ). Interestingly, the power decreases as the ICC increases, contrary to the established fact that power should be maintained at a level equal to or greater than the target level as the ICC increases (as it leads to an increase in the number of clusters required). This effect can be attributed to the conservative rounding of the number of clusters computed by SAS/GLMPOWER software. The power for a trial with correspondingly small number of clusters is more impacted by this rounding.

Table 4. Average power (SD) by coefficient of variation (CV) of cluster sizes and ICC ( $\rho$ )

\begin{tabular}{llcccccc}
\hline \multirow{2}{*}{$\mathrm{CV}$} & \multirow{2}{*}{$\begin{array}{l}\text { Method of } \\
\text { Analysis }\end{array}$} & $\begin{array}{c}\text { Type I } \\
\text { Error }\end{array}$ & Power & $\begin{array}{c}\text { Type I } \\
\text { error }\end{array}$ & Power & $\begin{array}{c}\text { Type I } \\
\text { error }\end{array}$ & Power \\
\hline & Equal & 0.048 & 0.76 & 0.051 & 0.74 & 0.051 & 0.77 \\
$0.30<\mathrm{CV}$ & Clusters & $(0.004)$ & $(0.13)$ & $(0.004)$ & $(0.06)$ & $(0.005)$ & $(0.03)$ \\
$\leq 0.50$ & & & & & & & \\
\hline
\end{tabular}




\begin{tabular}{llcccccc}
\hline \multirow{6}{*}{ Cluster Size } & 0.052 & 0.84 & 0.066 & 0.79 & 0.069 & 0.79 \\
& $(0.005)$ & $(0.08)$ & $(0.006)$ & $(0.02)$ & $(0.008)$ & $(0.01)$ \\
& & & & & & \\
& Minimum & 0.050 & 0.84 & 0.052 & 0.78 & 0.051 & 0.79 \\
& Variance & $(0.005)$ & $(0.08)$ & $(0.004)$ & $(0.03)$ & $(0.006)$ & $(0.01)$ \\
& & & & & & & \\
& Equal & 0.040 & 0.59 & 0.048 & 0.69 & 0.050 & 0.77 \\
& Clusters & $(0.010)$ & $(0.16)$ & $(0.004)$ & $(0.05)$ & $(0.007)$ & $(0.03)$ \\
& & & & & & & \\
CV $>0.50$ & Cluster Size & 0.060 & 0.83 & 0.086 & 0.79 & 0.086 & 0.78 \\
& & $(0.013)$ & $(0.08)$ & $(0.016)$ & $(0.02)$ & $(0.010)$ & $(0.01)$ \\
& & & & & & \\
& Minimum & 0.050 & 0.82 & 0.050 & 0.78 & 0.05 & 0.79 \\
& Variance & $(0.003)$ & $(0.08)$ & $(0.004)$ & $(0.02)$ & $(0.005)$ & $(0.02)$ \\
\hline
\end{tabular}

\section{DISCUSSION}

Sample size calculation is an important part of designing a trial, which may become a complicated task in a CRT when it is anticipated that there will be unequal clusters, due to the absence of an exact method, coupled with lack of knowledge of design parameters. This has led to approximate approaches that help overcome the loss in power due to imbalance in cluster sizes. Methods are available to inflate the sample size when the variability in cluster size is known a priori. However, the knowledge of distribution of cluster size may not be available at the time of planning a study. Further, many trials anticipating equal clusters end up with unequal clusters for a variety of reasons. Given these complications, the simplest approach is to calculate the sample size assuming equal cluster size, which may result in loss of trial power, given unequal cluster sizes in practice. Researchers may try to mitigate the impact of unequal clusters on trial power by performing a weighted analysis; however, there are several weights that may be employed there is a lack of consensus as to which method is best suited under certain circumstances. 
In this paper, we considered three methods of analysis for a CRT: assuming equal cluster sizes, weighted by cluster size, and weighted by minimum variance weights. Among these methods, we chose to focus on the one that allows us to make use of the straightforward analysis procedures of a GLM. We set out to investigate these methods under a variety of imbalance conditions, and to determine their effect on the type I error and power of the study. As such, we used a variety of distributions to generate the cluster sizes that should encompass a range of imbalances found in practice. We did not evaluate the bias of the estimates because the mean treatment effect remains the same regardless of whether an individual level analysis or a cluster level analysis is undertaken.(14)

We found that a simple analysis of cluster means assuming equal clusters yields satisfactory results as long as the variation in cluster size is low $(\mathrm{CV}<0.30)$. This result agrees with a prior result(5), where the authors concluded that variation in cluster size may be ignored if the $\mathrm{CV}$ of cluster size is less than 0.23 . As such, investigators observing low variation in cluster size may use a two-sample t-test to carry out their analysis. One example of such a case may be when classrooms within a school are randomized. A 2008 study by Salmon et al. examining the effectiveness of an intervention to prevent excess weight gain on children randomly assigned seventeen classes with approximately 25 children in each class to one of the three intervention groups or a control group.(23) In trials such as this, we expect to see minimal variation in cluster size from classroom to classroom within the same school. In situations where the cluster size variation is moderate to high, the approach fails to maintain the target power. 
We also examined a weighted approach to the final analysis, using both cluster size weights and minimum variance weights. A common approach among medical researchers is to use cluster size weights in the final analysis for its intuitive appeal that gives more weight to clusters of larger size.(14) However, we found the performance of this approach to be sub-par. Although the approach seems to maintain the power at target level, associated high levels of inflation in type I error may deem the conclusions questionable. Inflation in type I error becomes progressively worse as the variation in cluster size increase.

Among all weights, minimum variance weights have been found to be the best in terms of maintaining power of the trial by different researchers. $(1,14,17)$ Our simulation results support the claim that such weights perform the best. In addition, we present an explicit proof in the Appendix as to why such weights are optimal for an unbalanced cluster trial. Thus, we recommend using minimum variance weights in all situations where the cluster size variation is more than minimal.

Our results are consistent with those from others in the literature. In particular, our results are consistent with recommendations by Johnson et al, who also assessed type I error in CRTs across a variety of approaches, including weighting by cluster sizes, which was not among their recommend approaches(15). In addition, our results are similar to those obtained by van Breukelen et al in a series of manuscripts in which they incorporate the ICC and CV of the cluster size into the calculation of the sample size initially, and then assess results based on a variety of approaches. These papers further assess the utility of mixed models to account for clustering in the analysis, as do others.(24) 
It should be noted that individual level analyses are more efficient than using the cluster means(5); however, the use of cluster means allowed us to frame the problem in the context of the GLM. Hence, the results in this paper may not be applicable for other commonly used approaches to analyze data from CRTs (e.g., mixed model analysis, generalized estimating equations). With respect to sample size calculation, an alternate strategy for better design of CRT with unequal clusters makes use of $\mathrm{CV}$ of cluster sizes in the sample size formula(5) and adjusting the sample size using the relative efficiency of unequal to equal cluster size.( $(7,10)$ Application of such methods requires knowledge of the CV of cluster size and ICC, neither of which may available at the design stage of the study. We believe that these methods are better suited for sample size re-estimation procedures, where the knowledge gained in the initial phase of a trial is used to revisit the sample size calculation.

Another limitation of our study is the weighted t-test requires having a good prior estimate of intra-cluster correlation coefficient or between-cluster variability which is hard to obtain with a small number of clusters.(25) Johnson et al. found that the weighted t-test using the inverse of the estimated theoretical variance of the cluster means, with the variance components constrained to be positive, had the best Type I error distribution across all simulated scenarios, which can be performed with at least 6 clusters per arm and 16 participants per cluster.(15) Therefore, we do not recommend clinical trialists to use the weighted t-test when the number of clusters are less than 6 clusters per arm. When there are only 2 to 4 clusters per arm, neither individual-level nor hierachical model are sufficiently powered due to very limited degrees of freedom, and are not 
recommended except for pilot studies.(15) Finally, the methods described herein are only

appropriate when interest lies in evaluating the overall effectiveness of the intervention and not on making individual-level predictions, and only apply to normally distributed outcomes.

Discussions of approaches using mixed models, as well as for binary outcomes, can be found elsewhere. $(24,26)$

\section{Conclusions}

We provided a simple design and analysis procedure for CRTs with unequal clusters when the response variable is continuous. The methods are easy to implement using existing software, do not require advanced statistical knowledge of mixed effects model or population averaged model and are consistent with others' results.

\section{List of Abbreviations}

CRT: Cluster Randomized Trial

$\mathrm{CV}$ : Coefficient of Variation

EUBD: Exact Under Balanced Design

GLM: Generalized Linear model

ICC: Intraclass Correlation

\section{Ethics declarations}

\section{Ethics approval and consent to participate}

Not applicable. 


\section{Consent for publication}

Not applicable.

\section{Availability of data and materials}

Not applicable.

\section{Competing interests}

The authors declare that they have no competing interests.

\section{Funding}

Not applicable.

\section{Authors' contributions}

AR, LAM, CSC conceived of the problem; AR implemented the simulations and drafted the original draft; GS, LAM, CSC made critical revisions of the manuscript. All authors read and approved the final manuscript.

\section{Acknowledgements}

Not applicable.

\section{References}

1. Donner A, Klar N. Design and analysis of cluster randomization trials in health research. London: Arnold 2000. x, 178 p. p.

2. Hayes RJ, Alexander ND, Bennett S, Cousens SN. Design and analysis issues in clusterrandomized trials of interventions against infectious diseases. Statistical methods in medical research. 2000;9(2):95-116.

3. Huang SS, Septimus E, Kleinman K, Moody J, Hickok J, Avery TR, et al. Targeted versus universal decolonization to prevent ICU infection. The New England journal of medicine. 2013;368(24):2255-65.

4. Donner A, Birkett N, Buck C. Randomization by cluster. Sample size requirements and analysis. American Journal of Epidemiology. 1981;114(6):906-14.

5. Eldridge SM, Ashby D, Kerry S. Sample size for cluster randomized trials: effect of coefficient of variation of cluster size and analysis method. International Journal of Epidemiology. 2006;35(5):1292-300.

6. Manatunga AK, Hudgens MG, Chen S. Sample Size Estimation in Cluster Randomized Studies with Varying Cluster Size. Biometrical Journal. 2001;43(1):75-86.

7. van Breukelen GJ, Candel MJ, Berger MP. Relative efficiency of unequal versus equal cluster sizes in cluster randomized and multicentre trials. Statistics in Medicine. 2007;26(13):2589-603. 
8. van Breukelen GJ, Candel MJ. Calculating sample sizes for cluster randomized trials: we can keep it simple and efficient! Journal of clinical epidemiology. 2012;65(11):1212-8.

9. van Breukelen GJ, Candel MJ. Comments on 'Efficiency loss because of varying cluster size in cluster randomized trials is smaller than literature suggests'. Statistics in medicine. 2012;31(4):397-400.

10. You Z, Williams OD, Aban I, Kabagambe EK, Tiwari HK, Cutter G. Relative efficiency and sample size for cluster randomized trials with variable cluster sizes. Clinical trials. 2011;8(1):27-36.

11. Murray DM, Pals SL, Blitstein JL, Alfano CM, Lehman J. Design and analysis of grouprandomized trials in cancer: A review of current practices. J Natl Cancer I. 2008;100(7):483-91.

12. Muller KE, LaVange LM, Sharon Landesman R, Ramey CT. Power Calculations for General Linear Multivariate Models Including Repeated Measures Applications. Journal of the American Statistical Association. 1992;87(420):1209-26.

13. Gurka MJ, Coffey CS, Muller KE. Internal pilots for a class of linear mixed models with Gaussian and compound symmetric data. Statistics in Medicine. 2007;26(22):4083-99.

14. Kerry SM, Bland JM. Unequal cluster sizes for trials in English and Welsh general practice: implications for sample size calculations. Statistics in Medicine. 2001;20(3):377-90.

15. Johnson JL, Kreidler SM, Catellier DJ, Murray DM, Muller KE, Glueck DH. Recommendations for choosing an analysis method that controls Type I error for unbalanced cluster sample designs with Gaussian outcomes. Statistics in Medicine. 2015;34(27):3531-45.

16. Guittet L, Ravaud P, Giraudeau B. Planning a cluster randomized trial with unequal cluster sizes: practical issues involving continuous outcomes. BMC Medical Research Methodology. 2006;6:17.

17. Murray DM. Design and analysis of group-randomized trials. New York: Oxford University Press; 1998. xi, 467 p. p.

18. Kul S, Vanhaecht K, Panella M. Intraclass correlation coefficients for cluster randomized trials in care pathways and usual care: hospital treatment for heart failure. BMC health services research. 2014;14:84.

19. Smeeth $\mathrm{L}, \mathrm{Ng}$ ES. Intraclass correlation coefficients for cluster randomized trials in primary care: data from the MRC Trial of the Assessment and Management of Older People in the Community. Controlled clinical trials. 2002;23(4):409-21.

20. Clausson B, Lichtenstein P, Cnattingius S. Genetic influence on birthweight and gestational length determined by studies in offspring of twins. BJOG : an international journal of obstetrics and gynaecology. 2000;107(3):375-81.

21. Dong N, Reinke WM, Herman KC, Bradshaw CP, Murray DW. Meaningful Effect Sizes, Intraclass Correlations, and Proportions of Variance Explained by Covariates for Planning Twoand Three-Level Cluster Randomized Trials of Social and Behavioral Outcomes. Evaluation review. 2016;40(4):334-77.

22. Hunter DJ, Lange M, Snieder H, MacGregor AJ, Swaminathan R, Thakker RV, et al. Genetic contribution to renal function and electrolyte balance: a twin study. Clinical science. 2002;103(3):259-65.

23. Salmon J, Ball K, Hume C, Booth M, Crawford D. Outcomes of a group-randomized trial to prevent excess weight gain, reduce screen behaviours and promote physical activity in 10-yearold children: Switch-Play. International Journal of Obesity. 2008;32(4):601-12. 
24. Leyrat C, Morgan KE, Leurent B, Kahan BC. Cluster randomized trials with a small number of clusters: which analyses should be used? Int J Epidemiol. 2018;47(1):321-31.

25. Hayes RJ, Moulton LH. Cluster Randomised Trials. Second ed: CRC Press; 2017.

26. Candel MJ, Van Breukelen GJ. Sample size adjustments for varying cluster sizes in cluster randomized trials with binary outcomes analyzed with second-order PQL mixed logistic regression. Statistics in medicine. 2010;29(14):1488-501. 


\section{APPENDIX}

Theorem 1: For a CRT with unequal cluster sizes, the linear mixed model may be equivalently expressed as a weighted univariate linear model that can provide exact inference when the weights are completely known. The approach provides approximate estimates when the weights need to be estimated from the data, which is the case for unbalanced cluster trails.

(1) As described in Section 2.1, a linear mixed model for $p_{g i}$ - correlated observations on a cluster $i \in\left\{1, \ldots, N_{2}\right\} \quad$ may $\quad$ be written $\quad$ as $\quad \boldsymbol{y}_{i}=\boldsymbol{X}_{i} \boldsymbol{\beta}+\boldsymbol{z}_{i} d_{i}+\boldsymbol{e}_{i} \quad$ with $\quad E\left(\boldsymbol{y}_{i}\right)=\boldsymbol{X}_{i} \boldsymbol{\beta} \quad$ and $\mathcal{V}\left(\boldsymbol{y}_{i}\right)=\boldsymbol{\Sigma}_{i}=\sigma_{b}^{2} \boldsymbol{z}_{i} \boldsymbol{z}_{i}^{\prime}+\sigma_{e}^{2} \boldsymbol{I}_{p_{g i}}$. With only one random effect for cluster, $\boldsymbol{z}_{i} \equiv \mathbf{1}_{p_{g i}}$, which leads to $\boldsymbol{\Sigma}_{i}=\sigma_{b}^{2} \mathbf{1}_{p_{g i}} \mathbf{1}_{p_{g i}}^{\prime}+\sigma_{e}^{2} \boldsymbol{I}_{p_{g i}}$. In terms of the total variance of the outcome variable, the covariance matrix is $\boldsymbol{\Sigma}_{i}=\sigma^{2}\left[\mathbf{1}_{p_{g i}} \mathbf{1}_{p_{g i}}^{\prime} \rho+\boldsymbol{I}_{p_{g i}}(1-\rho)\right]$. Thus, $\boldsymbol{y}_{i} \sim \mathcal{N}_{p_{g i}}\left(\boldsymbol{X}_{i} \boldsymbol{\beta}, \boldsymbol{\Sigma}_{i}\right)$.

(2) The data from all the clusters may be "stacked" to represent a combined model. With a total of $n$ observations, the model can be succinctly written as $\boldsymbol{y}_{s}=\boldsymbol{X}_{s} \boldsymbol{\beta}+\boldsymbol{Z}_{s} \boldsymbol{d}+\boldsymbol{e}_{s}$.

(3) $\boldsymbol{y}_{s} \sim \mathcal{N}_{n}\left(\boldsymbol{X}_{s} \boldsymbol{\beta}, \boldsymbol{\Sigma}_{s}\right)$ where $\boldsymbol{\Sigma}_{s}=\sigma^{2} \bigoplus_{i=1}^{N_{2}}\left[\mathbf{1}_{p_{g i}} \mathbf{1}_{p_{g i}}^{\prime} \rho+\boldsymbol{I}_{p_{g i}}(1-\rho)\right]$ is of the order $n \times n$. This can be realized by using cell mean coding for the design matrix $\boldsymbol{X}_{s}=D g\left(\mathbf{1}_{n_{1}}, \ldots, \mathbf{1}_{n_{G}}\right)$ and with only a single random effect, $\boldsymbol{Z}_{s}=D g\left(\mathbf{1}_{p_{g 1}}, \ldots, \mathbf{1}_{p_{g N_{2}}}\right)$. (Note: $\bigoplus$ is a direct sum operator)

(4) Pre-multiplying the stacked model by a $N_{2} \times n$ matrix of known constants, $\boldsymbol{T}$, yields a vector of cluster means. Hence, the new model is given by:

$$
\underset{\left(N_{2} \times 1\right)}{\boldsymbol{T} \boldsymbol{y}_{s}}=\underset{\left(N_{2} \times G\right)}{\left(\boldsymbol{T} \boldsymbol{X}_{s}\right)} \boldsymbol{\beta}+\underset{\left(N_{2} \times N\right)}{\left(\boldsymbol{T} \boldsymbol{Z}_{s}\right)} \boldsymbol{d}+\underset{\left(N_{2} \times 1\right)}{\boldsymbol{T} \boldsymbol{e}_{s}}
$$

The model in (A1) is equivalent to the following model in (A2) with $\boldsymbol{y}=\boldsymbol{T} \boldsymbol{y}_{s}, \boldsymbol{X}=\boldsymbol{T} \boldsymbol{X}_{s}$, $Z=T Z_{s}$ and $e=T e_{s}$

$$
y=X \beta+Z d+e
$$


With this model, we still have $\boldsymbol{d} \sim \mathcal{N}_{N_{2}}\left(\mathbf{0}, \sigma_{b}^{2} \boldsymbol{I}_{N_{2}}\right)$ mutually independent of $\boldsymbol{e}=\boldsymbol{T} \boldsymbol{e}_{s}$ but with the transformation, $\boldsymbol{e} \sim \mathcal{N}\left(\mathbf{0}, \sigma_{e}^{2} \boldsymbol{T} \boldsymbol{T}^{\prime}\right)$ where $\sigma_{e}^{2} \boldsymbol{T} \boldsymbol{T}^{\prime}=\sigma_{e}^{2} \cdot D g\left(1 / p_{g 1}, \ldots, 1 / p_{g N_{2}}\right)$. Therefore, the $N_{2} \times N_{2}$ covariance matrix is given by $\mathcal{V}(\boldsymbol{y})=\boldsymbol{\Sigma}=\operatorname{Diag}\left(\sigma_{b}^{2}+\sigma_{e}^{2} / p_{g 1}, \ldots, \sigma_{b}^{2}+\sigma_{e}^{2} / p_{g N_{2}}\right)$ which can be shown to be equivalent to $\boldsymbol{\Sigma}=\sigma^{2} \cdot \operatorname{Diag}\left(\left[1+\left(p_{g 1}-1\right) \rho\right] / p_{g 1}, \ldots,\left[1+\left(p_{g N_{2}}-1\right) \rho\right] / p_{g N_{2}}\right)=\sigma^{2} \boldsymbol{D}$.

Thus, $\boldsymbol{y} \sim \mathcal{N}_{N_{2}}\left(\boldsymbol{X} \boldsymbol{\beta}, \boldsymbol{\Sigma}=\sigma^{2} \boldsymbol{D}\right)$. This is a GLM with heteroscedastic errors. If all the cluster sizes are equal then the model reduces to a GLM with independent errors. For a CRT with unequal cluster sizes, the $\boldsymbol{D}$ matrix is a function of ICC and cluster size. Examining the elements of the matrix, it can be seen that the elements are in fact the minimum variance weights. Thus using estimates of such weights have the optimal properties for a CRT with unequal sizes when a weighted analysis is performed. 


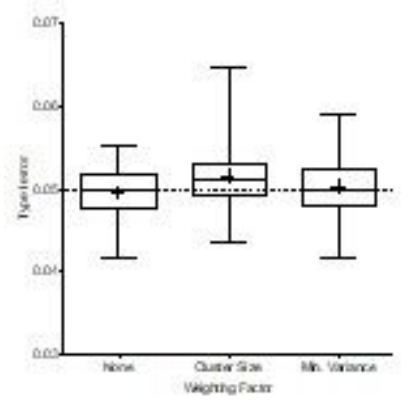

(a)



(b)
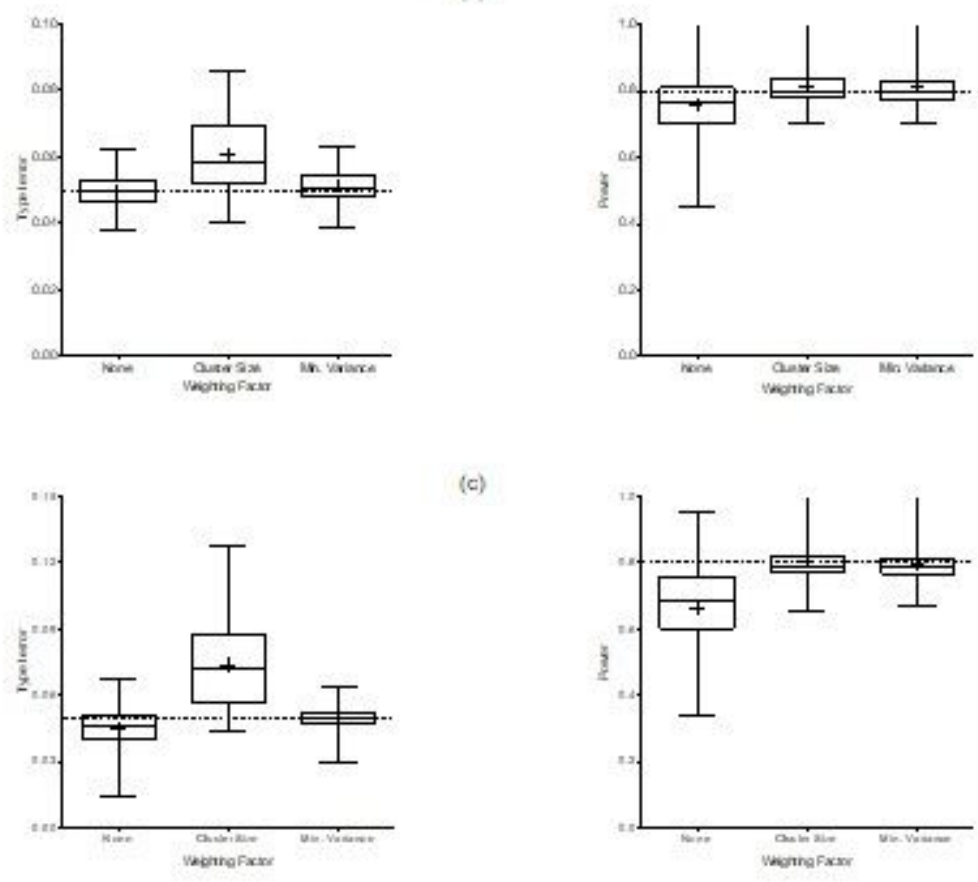

(c)

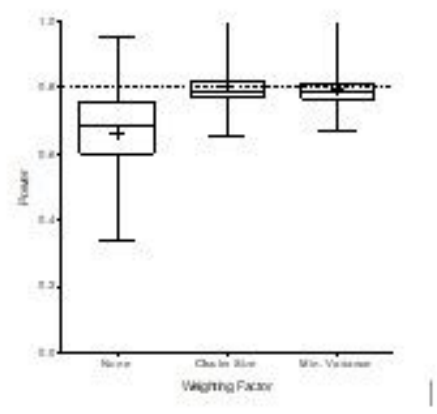

\section{Figure 1}

Distribution of type I error (left) and power (right) for three categories of CV Distribution of type I error (left) and power (right) for the three categories of CV: (a) (b) and (c). The reference line for nominal type I error and target power are shown by a dotted line at 0.05 and 0.80 respectively. 